\title{
The destruction and survival of polycyclic aromatic hydrocarbons in the disks of $T$ Tauri stars
}

\author{
R. Siebenmorgen ${ }^{1}$ and E. Krügel ${ }^{2}$ \\ ${ }^{1}$ European Southern Observatory, Karl-Schwarzschild-Str. 2, 85748 Garching b. München, Germany \\ e-mail: rsiebenm@eso.org \\ 2 Max-Planck-Institut für Radioastronomie, Auf dem Hügel 69, Postfach 2024, 53010 Bonn, Germany
}

Received 10 March 2009 / Accepted 4 December 2009

\begin{abstract}
In Spitzer observations of Tauri stars and their disks, features of polycyclic aromatic hydrocarbons (PAHs) are detected in less than $10 \%$ of the objects, although the stellar photosphere is sufficiently hot to excite PAHs. To explain the deficiency, we discuss PAH destruction by photons, assuming that the star has beside its photospheric emission also a far ultraviolet (FUV), an extreme ultraviolet (EUV) and an X-ray component with a fractional luminosity of $1 \%, 0.1 \%$ and $0.025 \%$, respectively. We consider as a PAH destruction process unimolecular dissociation and present a simplified scheme to estimate the location from the star at which the molecules become photo-stable. We find that soft photons with energies below $\sim 20 \mathrm{eV}$ dissociate PAHs only up to short distances from the star $(r<1 \mathrm{AU})$; whereas dissociation by hard photons (EUV and X-ray) is so efficient that it would destroy all PAHs (from regions in the disk where they could be excited). As a possible path for PAH-survival we suggest turbulent motions in the disk. They can replenish or remove PAHs from the reach of hard photons. For standard disk models, where the surface density changes like $r^{-1}$ and the mid plane temperature like $r^{-0.5}$, the critical vertical velocity for PAH survival is proportional to $r^{-3 / 4}$ and equals $\sim 5 \mathrm{~m} / \mathrm{s}$ at $10 \mathrm{AU}$, which is in the range of expected velocities in the surface layer. The uncertainty in the parameters is large enough to explain both detection and non-detection of PAHs. Our approximate treatment also takes into account the presence of gas which is ionized at the top of the disk and neutral at lower levels.
\end{abstract}

Key words. protoplanetary disks - dust, extinction - infrared: stars - X-rays: stars - X-rays: ISM - radiation mechanisms: general

\section{Introduction}

Infrared emission bands of PAHs can be used as a probe of the UV environment. They are commonly seen in the interstellar medium (ISM), but also in young stellar objects such as Herbig Ae/Be stars (Waelkens et al. 1996; Siebenmorgen et al. 2000; Meeus et al. 2001; Peeters et al. 2002; van Boekel et al. 2004). The observed emission can be explained in models of an irradiated disk (Habart et al. 2004; Visser et al. 2007; Dullemond et al. 2007a).

The infrared space observatory (ISO) looked at a few of the much fainter T Tauri stars, but without a clear PAH detection (Siebenmorgen et al. 2000). In the Evans et al. (2003) legacy program, which employs the more sensitive Spitzer Space Telescope (SST), three out of $38 \mathrm{~T}$ Tauri stars show PAH features (Geers et al. 2006). This corresponds to a detection rate of only $8 \%$ in contrast to almost $60 \%$ found in Herbig Ae/Be stars (Acke $\&$ van den Ancker 2004). Similarly low rates for T Tauri stars are found by Furlan et al. (2006) who present 111 SST spectra in the Taurus-Auriga star forming region and speculate that the absence of PAH resonances is due to the much weaker UV field compared to Herbig Ae/Be stars. Geers et al. (2009), on the other hand, argue that the PAHs are simply under-abundant relative to the ISM. They also find that variations of the disk geometry, such as flaring or gaps, have only a small effect on the strength of the PAH bands. Clearing out gas and dust by planet formation inside the disk could effectively remove PAHs. Indeed, inner gaps in disks are observed at radii between 40-60 AU and at wavelengths between $20-1000 \mu \mathrm{m}$, where the emission is dominated by large grains (Brown et al. 2008; Geers et al. 2007b). But in cases where PAH emission is resolved, it is extended up to 15-60 AU without sub-structure and inside the inner gap region (Geers et al. 2007a). The spatial extent of the PAH emission is similar for T Tauri as well as Herbig Ae/Be stars. We therefore suggest that PAH removal by radiative destruction is dominant.

Present radiative transfer models of the PAH emission from dusty disks consider only the stellar radiation field and no additional EUV or X-ray component (Habart et al. 2004; Geers et al. 2006; Visser et al. 2007; Dullemond et al. 2007a). Their hard photons could, according to laboratory experiments (Ruhl et al. 1989; Leach et al. 1989a,b; Jochims et al. 1994) and theory (Omont 1986; Tielens 2005; Rapacioli et al. 2006; Micelotta et al. 2010a,b), destroy PAHs. We discuss below their impact on the PAH abundance in the disks of T Tauri stars.

\section{Radiation components of $T$ Tauri stars}

Our T Tauri model star has a total luminosity $L_{*}=2 L_{\odot}$. Its radiation consists of a photospheric, a FUV, an EUV and an X-ray component. Their parameters are listed in Table 1 and are very similar to those proposed by Gorti \& Hollenbach (2008). The total spectrum is displayed in Fig. 1. We point out that the FUV and EUV radiation are observationally poorly constrained.

The photosphere supplies most of the luminosity, whereas the FUV, EUV and X-ray radiation are much weaker and believed to originate from accretion onto the star as well as from chromospheric and coronal activity. The photosphere, the 
Table 1. The four radiation components of our T Tauri model star.

\begin{tabular}{|c|c|c|c|c|c|}
\hline & & (1) & (2) & (3) & (4) \\
\hline$i$ & component & $L / L_{*}$ & spectrum & $\begin{array}{l}h \bar{\nu}_{\mathrm{em}} \\
(\mathrm{eV})\end{array}$ & $\begin{array}{c}F_{10} \\
\left(\mathrm{erg} \mathrm{s}^{-1} \mathrm{~cm}^{-2}\right)\end{array}$ \\
\hline 1 & photosphere & 0.99 & $4000 \mathrm{~K} \mathrm{BB}$ & 0.9 & 30000 \\
\hline 2 & FUV & 0.01 & $15000 \mathrm{~K} \mathrm{BB}$ & 3.5 & 300 \\
\hline 3 & EUV & 0.001 & $3 \times 10^{5} \mathrm{~K} \mathrm{BB}$ & 70 & 30 \\
\hline 4 & $\mathrm{X}$-rays $(h v<2 \mathrm{keV})$ & $2.5 \times 10^{-4}$ & $\propto v^{2}$ & 1330 & 10 \\
\hline
\end{tabular}

Notes. (1) fractional luminosity; (2) spectral shape (BB = blackbody); (3) mean energy of emitted photons, $h \bar{v}_{\text {em }}$; (4) approximate flux at $10 \mathrm{AU}, F_{10}$.

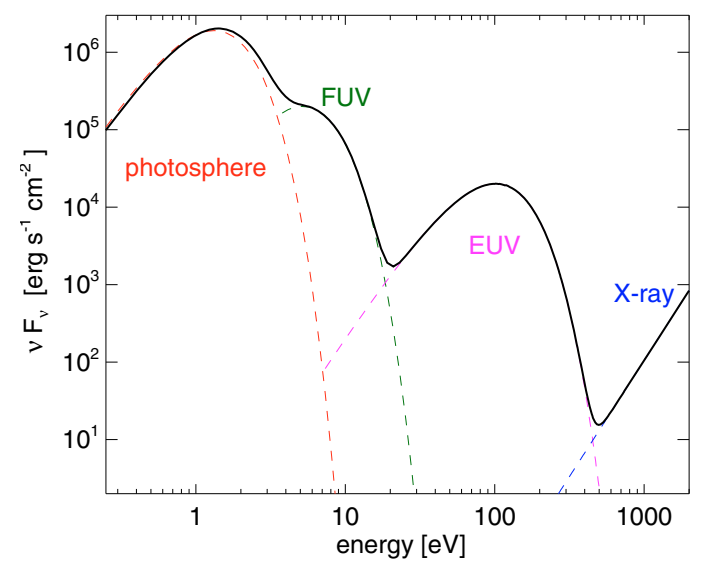

Fig. 1. The spectral energy distribution of our T Tauri model star at $1 \mathrm{AU}$ without foreground extinction (Eq. (1)). The absolute luminosities of the components are given in Table 1.

FUV and EUV component are approximated by blackbodies. We assume $4000 \mathrm{~K}$ for the photosphere and, following Stahler et al. (1980) and Calvet \& Gullbring (1998), $15000 \mathrm{~K}$ for the FUV (pre-shock) and $\sim 3 \times 10^{5} \mathrm{~K}$ for the EUV emission (postshock region).

The strength of the FUV and EUV radiation is determined by the accretion luminosity, which we approximate by $L_{\text {acc }}=$ $G M_{*} \dot{M} / R_{*}$. If $R_{*}=2 R_{\odot}$ and $M_{*}=1 M_{\odot}$ are the radius and mass of the star, an accretion rate $\dot{M}=10^{-9} M_{\odot} \mathrm{yr}^{-1}$ (Akeson et al. $2005)$ yields $L_{\text {acc }} \sim 0.01 L_{*}$. Higher values $\left(L_{\text {acc }} / L_{*} \gtrsim 0.1\right)$, but with a large spread, are derived by Muzerolle et al. (1998, 2003) from hydrogen emission lines. However, as we show in Sect. 5, such stronger fluxes have little influence on the stability analysis of PAHs.

Preibisch et al. (2006) establish from Chandra observations $(0.5-8 \mathrm{keV})$ a relation between the X-ray luminosity $L_{\mathrm{x}}$ and the total luminosity $L_{*}$, confirming the ROSAT results of Sterzik \& Schmitt (1997). The ratio $L_{\mathrm{x}} / L_{*}$ is similar in rapidly rotating main-sequence stars and non-accreting $\mathrm{T}$ Tauri stars $\left(\sim 10^{-3}\right)$, but systematically lower by a factor of about four in accreting T Tauri stars (Preibisch et al. 2006). Interestingly, $L_{\mathrm{x}} / L_{*}$ is much smaller $\left(\sim 10^{-7} \ldots 10^{-5}\right.$, Stelzer et al. 2006) in Herbig $\mathrm{Ae} / \mathrm{Be}$ stars, with values comparable to those of the Sun. The solar X-ray luminosity in the $0.1-2.4 \mathrm{keV}$ ROSAT passband lies in the range of $10^{-6.8} \lesssim L_{\mathrm{x}} / L_{*} \lesssim 10^{-5.7}$ during a solar cycle and is typical for G stars (Judge et al. 2003).

X-ray fluxes are generally variable on timescales of hours to weeks and weaken during the evolution of the $\mathrm{T}$ Tauri star. For example, half of the sources in the Taurus molecular cloud detected by XMM/Newton $(0.3-7.8 \mathrm{keV})$ show variations, more often at hard $(>0.5 \mathrm{keV})$ than at soft energies, and a quarter of them display flares (Stelzer et al. 2007) about once a week and lasting for a few hours. In a strong flare, more than $10^{35} \mathrm{erg}$ are emitted, and $L_{\mathrm{x}}$ can reach $1 \%$ of the total luminosity. We assume up to $2 \mathrm{keV}$ a power law spectrum $\propto v^{2}$ (Güdel et al. 2007) and neglect harder radiation, because the emission then steeply declines $\left(\propto v^{-3}\right)$.

Let $L_{i}$ be the frequency-integrated luminosity of the radiation component $i$ (see Table 1) and $L_{i, v}$ its spectral luminosity, so that $L_{i}=\int L_{i, v} \mathrm{~d} v$. Dropping the index $i$ for convenience, the flux (of the component $i$ ) at a distance $r$ is

$F_{v}=\frac{L_{v} \mathrm{e}^{-\tau_{v}}}{4 \pi r^{2}}$.

We include a screening factor $\mathrm{e}^{-\tau_{\nu}}$ to account for foreground absorption (by dust and gas). If $\kappa_{v}$ denotes the absorption cross section per carbon atom, a PAH of $N_{\mathrm{c}}$ carbon atoms absorbs in one second (from the component $i$ )

$N_{\gamma}=N_{\mathrm{c}} \int \frac{F_{v} K_{v}}{h v} \mathrm{~d} v$

photons of total energy

$E_{\mathrm{abs}}=N_{\mathrm{c}} \int F_{v} \kappa_{v} \mathrm{~d} v$

The inverse of $N_{\gamma}$ is the average time between two absorption events,

$t_{\mathrm{abs}}=N_{\gamma}^{-1}$.

The mean photon energy equals

$h \bar{v}=\frac{\int F_{v} K_{v} \mathrm{~d} v}{\int \frac{F_{v} K_{v}}{h v} \mathrm{~d} v}$.

\section{Cross sections}

As the light from the star enters the disk, it is attenuated by gas and dust. The absorption cross section of gas depends on the ionization stage of the atoms, which is determined by the balance between recombination and photo-ionization. By far the most important atoms are, of course, hydrogen and helium with ionization potentials of $13.6 \mathrm{eV}$ and $24.6 \mathrm{eV}$, respectively. Because the recombination rate is proportional to the square of the gas density, which is high in the disk (Sect. 5), the gas is ionized only in a thin surface layer $\left(A_{V}<0.001 \mathrm{mag}\right.$, Sect. 2.6 of Gorti \& Hollenbach 2008). We use the atomic cross sections of Morrison \& McCommon (1982) and Balucinska-Church \& McCommon (1992) as well as solar element abundances. 


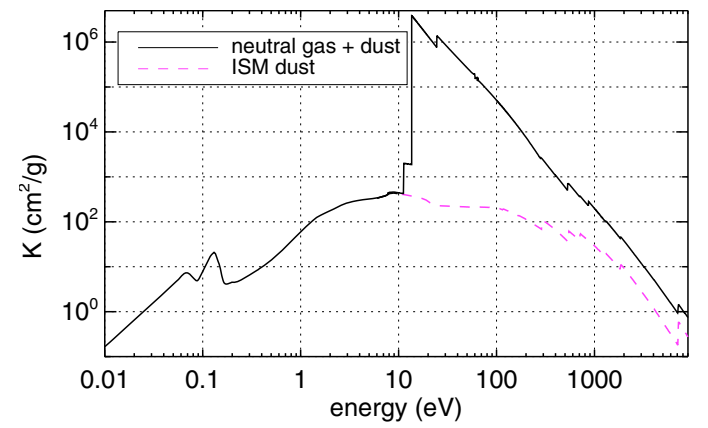

Fig. 2. The mass extinction coefficient per gram disk material when the gas is neutral (Morrison \& McCommon 1983); the gas-to-dust mass ratio equals 130 .

The dust cross sections are taken from the model of Krügel (2006), which describes standard dust. The absorption efficiency calculated from the Mie theory must be corrected downwards for X-rays. Hard photons can eject electrons from the grain, and as these conduct kinetic energy, only part of the photon energy is deposited in the dust particle. The threshold $E_{\mathrm{t}}$, above which such a correction is necessary, depends on the grain size; details are given in Dwek \& Smith (1996). For a $10 \AA$ graphite particle, $E_{\mathrm{t}} \sim 100 \mathrm{eV}$, and the reduction factor is roughly proportional to $v^{-1}$.

The absorption coefficient of dust $K_{\mathrm{d}, \lambda}$ and of neutral gas plus dust $K_{\lambda}=K_{\mathrm{gas}, \lambda}+K_{\mathrm{d}, \lambda}$, both per gram of disk material, are plotted in Fig. 2 for a dust-to-gas mass ratio of 1:130. Note that at the ionization threshold of hydrogen, $K_{\text {gas }}$ is almost $10^{4}$ times greater than $K_{\mathrm{d}}$.

With respect to the absorption cross section of PAHs, we assume $\kappa_{v}=7 \times 10^{-18} \mathrm{~cm}^{2}$ per carbon atom when $h v<13.6 \mathrm{eV}$, and scale $\kappa_{v}$ at higher energies to follow the values of a graphite sphere of $10 \AA$ radius (Dwek \& Smith 1996). The maximum wavelength (in $\AA$ ) for the PAH excitation is $\lambda_{\text {max }, \mathrm{PAH}}=1630+$ $370 \sqrt{N_{\mathrm{c}}}$ (Schutte et al. 1993), resulting in a minimum photon energy of $2.3 \mathrm{eV}$ for a PAH with $N_{\mathrm{c}}=100$ carbon atoms.

\subsection{PAH emission}

As the PAHs are transiently heated, their excitation is usually treated statistically. For the computation we follow the method of Guhathakurta \& Draine (1989) who consider a large ensemble of PAHs in a steady state. Let $P(T) \mathrm{d} T$ be the probability of finding an arbitrary PAH in the temperature interval $[T, T+\mathrm{d} T]$. The temperature distribution $P(T)$ is calculated in this method from a transition matrix $\left(A_{\mathrm{fi}}\right)$. The PAH absorption cross section is denoted by $K_{v}$. The matrix element $A_{\mathrm{fi}}$ refers to dust heating from an initial enthalpy bin centered at $U_{\mathrm{i}}$ to a final one centered at $U_{\mathrm{f}}$. The final enthalpy bin is of a width of $\Delta U_{\mathrm{f}}$. For a mono-chromatic flux, the transition matrix equals to

$A_{\mathrm{fi}}=\left\{\begin{array}{cl}\frac{K_{v} F}{h v} & : \text { if }\left|U_{\mathrm{f}}-U_{\mathrm{i}}-h v\right| \leq \frac{1}{2} \Delta U_{\mathrm{f}} . \\ 0 & : \text { else }\end{array}\right.$

Examples of $P(T)$ are displayed in Fig. 3 for mono-chromatic fluxes, which cover almost the entire range encountered anywhere in the disk in terms of intensity, the flux is varied from $F=10$ to $10^{7} \mathrm{erg} \mathrm{s}^{-1} \mathrm{~cm}^{-2}$, and also the hardness, the photon energy is between $h v=3.8 \mathrm{eV}$ and $1 \mathrm{keV}$. The approximate unattenuated fluxes of the four radiation components at a distance of $10 \mathrm{AU}$ are listed in Table 1 . Note that when $F=n_{\gamma} h v$ is constant, the number of photons $n_{\gamma}$ decreases as the photon energy

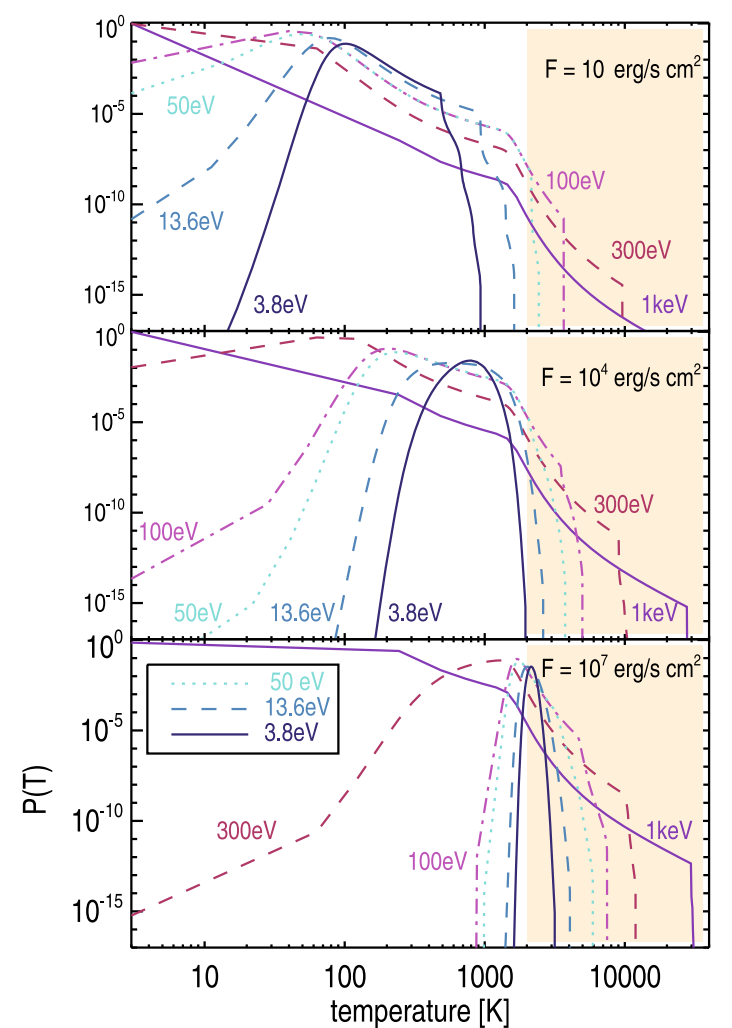

Fig. 3. The temperature distribution $P(T)$ of a PAH with $100 \mathrm{C}$ atoms exposed to mono-chromatic radiation with $h v=3.8,13.6,50,100,300 \mathrm{eV}$ and $1 \mathrm{keV}$. This set includes the mean photon energies of the four radiation components of the T Tauri star. The fluxes range from (top to bottom) $F=10$ to $10^{7} \mathrm{erg} \mathrm{s}^{-1} \mathrm{~cm}^{-2}$. The shaded area marks temperatures above the sublimation temperature of graphite.

$h v$ increases. The power absorbed by one PAH is almost independent of the photon energy as long as $h v \lesssim 100 \mathrm{eV}$ (see Fig. 4). By and large, when $h v$ is fixed and $F$ increases, the curves in Fig. 3 narrow and move to the right towards higher temperatures. When the radiation field is weak $\left(F=10 \mathrm{erg} \mathrm{s}^{-1} \mathrm{~cm}^{-2}\right)$ and the photons are soft $(h v=3.8 \mathrm{eV})$, the PAH absorbs about one photon per day, and there is plenty of time to cool down. In this case, the PAH virtually never exceeds the sublimation temperature $T_{\mathrm{s}}$, (at which solid carbon gasifies). For a wide pressure range $\left(10^{-1}-10^{-7} \mathrm{dyn} \mathrm{cm}^{-2}\right), T_{\mathrm{s}} \sim 2000 \mathrm{~K}$ for graphite (CRC Handbook of Chemistry \& Physics 2005; Salpeter et al. 1977). In Fig. 3 we highlight the area where the temperature is above $T_{\mathrm{s}}$. For a PAH exposed to a strong radiation field $\left(F=10^{7} \mathrm{erg} \mathrm{s}^{-1} \mathrm{~cm}^{-2}\right)$, about a dozen soft photons are absorbed within a cooling time, and the temperature distribution function becomes very narrow around $2000 \mathrm{~K}$, and sublimation is likely. For hard photons $(h v \geq 50 \mathrm{eV})$, the PAH undergoes extreme temperature excursions independent of the strength of the radiation field. This indicates that PAH become photo-unstable either by absorption of a single hard photon, or by soft photons, if there are many of them.

\section{PAH destruction}

The abundance of PAHs is determined by the competition between the formation and destruction processes under the specific environmental conditions. Underlying processes are discussed, for example, by Omont (1986), Voit (1992), or recently 


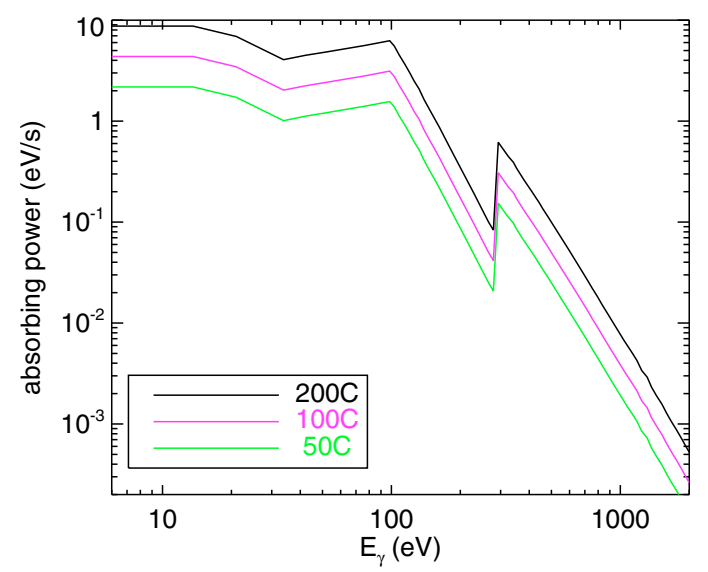

Fig. 4. The power $W=N_{\mathrm{c}} \kappa F$ absorbed by one PAH, with the number of $\mathrm{C}$ atoms $N_{\mathrm{C}}$ as indicated, in a mono-chromatic flux $F=$ $10^{4} \mathrm{erg} \mathrm{s}^{-1} \mathrm{~cm}^{-2}$ as a function of the photon energy $E_{\gamma}=h v$.

by Micelotta et al. (2010a). Here we only consider PAH destruction by photons and generally assume that PAH formation is negligible. After photon absorption, a highly vibrationally excited PAH may relax through emission of IR photons or, if sufficiently excited, lose atoms. The latter process is called unimolecular dissociation and is discussed for interstellar PAHs by Allamandola (1989), Leger et al. (1989), Le Page et al. (2003), Rapacioli et al. (2006), and Micelotta et al. (2010b). Laboratory studies of PAH dissociation which can be applied to astrophysical situations are rare (Jochims et al. 1994). The photo-chemistry of PAHs is reviewed by Tielens $(2005,2008)$.

\subsection{Procedure}

In the disks of $\mathrm{T}$ Tau stars, the PAH abundance obviously depends on place and on time as the disk evolves. There is no general solution to the problem, and to extract numbers, we have to radically simplify it. We wish to find some estimate of the location where PAHs become stable against photo-destruction. To derive a procedure, we recall that although after absorption of an energetic photon its energy is immediately distributed over all available vibrational modes (Allamandola et al. 1989), the excitation of a particular atom fluctuates, and it is occasionally pushed into the continuum and leaves the PAH. Quantitatively, the unimolecular dissociation can be written in the Arrhenius form. In a classical description, an atom of a critical (Arrhenius) energy $E_{0}$ detaches from a PAH of a peak temperature $T_{\mathrm{p}}$ if the dissociation time

$t_{\text {dis }} \sim v_{0}^{-1} \mathrm{e}^{E_{0} / k T_{\mathrm{p}}}$

is shorter than the cooling time $t_{\text {cool }}$. A characteristic value for the vibrational frequency is $v_{0} \sim 10^{13} \mathrm{~s}^{-1}$. The "atom", which may apart from $\mathrm{H}$ or $\mathrm{C}$ also be an atomic group like $\mathrm{C}_{2} \mathrm{H}_{2}$, needs the time $t_{\text {dis }}$ to overcome the critical internal barrier $E_{0}$, which is similar but not identical to the chemical binding energy. Micelotta et al. (2010b) quote $E_{0}$ of $3.2 \mathrm{eV}$ for H-loss, $4.2 \mathrm{eV}$ for $\mathrm{C}_{2} \mathrm{H}_{2}, 7.5 \mathrm{eV}$ for pure $\mathrm{C}$ loss and $9.5 \mathrm{eV}$ for $\mathrm{C}_{2}$. For the ISM they find $E_{0}=4.6 \mathrm{eV}$ and a somewhat larger value for photo dissociation regions. The inverse of the dissociation time is the probability that a certain atom leaves the PAH per unit time.

The exponential term $\mathrm{e}^{E_{0} / k T_{\mathrm{p}}}$ in Eq. (7) increases very rapidly as $T$ falls, and meaningful values (i.e. not too large ones) of $t_{\text {dis }}$ are obtained only if $T_{\mathrm{p}}>1500 \mathrm{~K}$. Atoms will only detach when $t_{\text {dis }}<t_{\text {cool }}$. As the cooling time at these temperatures is for astrophysical applications on the order of $1 \mathrm{~s}$, independent of the PAH size, the dissociation criterion reads

$t_{\text {dis }} \lesssim 1 \mathrm{~s}$

It leads to a minimum temperature for destruction

$T_{\text {dis }}=\frac{E_{0}}{k \ln v_{0}}$

Assuming $E_{0} \sim 5 \mathrm{eV}$, one gets $T_{\text {dis }} \simeq 2000 \mathrm{~K}$. At this high temperature, the internal energy of a PAH is reasonably well approximated by $3 N_{\mathrm{c}} k T_{\text {dis }}$ if one also takes the presence of H-modes into account. The minimum temperature $T_{\mathrm{dis}}$ is related to a minimum energy input $\Delta E$. The PAH is therefore unstable to photons with

$\Delta E \geq h v_{\mathrm{c}}=3 N_{\mathrm{c}} k T_{\mathrm{dis}}=\frac{3}{\ln v_{0}} N_{\mathrm{c}} E_{0} \simeq 0.1 N_{\mathrm{c}} E_{0}$,

or when the number of carbon atoms

$N_{\mathrm{c}} \leq \frac{2 \Delta E}{[\mathrm{eV}]}$

Micelotta et al. (2010b) find that a PAH with $N_{\mathrm{c}}=50$ requires an internal energy of about $\Delta E=24 \mathrm{eV}$ to dissociate, which agrees with the above estimate (Eq. (11)).

The minimum energy input required for dissociation can either be delivered by absorption of $i$ ) many soft photons with a total energy $E_{\mathrm{abs}} \geq \Delta E$ (Eq. (3)); or $i i$ ) by a single hard photon, with an energy $h v \geq \Delta E$. If a photon heats the PAH to a peak temperature much above $T_{\mathrm{dis}}$, more than one atom will detach. The first expulsion occurs momentarily $\left(t_{\text {dis }} \ll 1 \mathrm{~s}\right)$. It consumes the energy $E_{0}$ plus some kinetic energy $E_{\text {kin }}$ for the liberated atom. The new PAH temperature follows from

$h v-E_{0}-E_{\text {kin }}=3\left(N_{\mathrm{c}}-1\right) k T$.

This happens $x$ times until $T$ has dropped to $T_{\text {dis }}$,

$h v-x\left(E_{0}+E_{\text {kin }}\right)=3\left(N_{\mathrm{c}}-x\right) k T_{\text {dis }}$.

With $E_{\text {kin }} \sim 0.5 \mathrm{eV}$ we estimate that the total number of freed atoms is

$x=\frac{h v-3 N_{\mathrm{c}} k T_{\mathrm{dis}}}{E_{0}+E_{\mathrm{kin}}-3 k T_{\mathrm{dis}}} \simeq \frac{h v}{5[\mathrm{eV}]}-\frac{N_{\mathrm{c}}}{10}$.

For $N_{\mathrm{c}}=100$, an average EUV photon ejects nine atoms, and one X-ray photon destroys the whole PAH (Col. (5) in Table 2).

\subsection{Disruption by Coulomb forces}

For completeness we also mention the possible disruption of PAHs by Coulomb forces. Double or multiple ionization of a PAH loosens the binding of the peripheral $\mathrm{H}$ atoms as well as that of the skeleton of carbon atoms. The ejection of K-shell electrons by X-ray photons $(h v>284 \mathrm{eV})$ in combination with Auger electrons will amplify the process. Coulomb explosion is relevant mainly for small PAHs and is neglected here. 
Table 2. Quantities relevant to PAH survival.

\begin{tabular}{crcccccccr}
\hline \hline & $(1)$ & $(2)$ & $(3)$ & $(4)$ & $(5)$ & $(6)$ & $(7)$ & $(8)$ & $(9)$ \\
\hline component & $h \bar{v}$ & $\kappa$ & $\eta$ & $K / K_{\mathrm{V}}$ & $x$ & $z_{0} / H$ & $\ell / H$ & $t_{\text {abs }}$ & $v_{\text {cr }}$ \\
& $\mathrm{eV}$ & $10^{-4} \AA^{2}$ & & & & & & & $\mathrm{~m} / \mathrm{s}$ \\
photosphere & 2.7 & 700 & $\ll 1$ & 1.2 & - & 4.4 & 0.5 & $5 \mathrm{~s}$ & - \\
FUV & 4.2 & 700 & $\ll 1$ & 1.5 & - & 4.5 & 0.5 & $120 \mathrm{~s}$ & - \\
EUV & 97 & 36 & $\sim 0.5$ & 110 & 9 & 5.3 & 0.4 & 9 days & 2900 \\
X-ray & 1100 & 0.17 & $\ll 1$ & 0.55 & 100 & 4.2 & 0.5 & $290 \mathrm{yr}$ & 3.4 \\
\hline
\end{tabular}

Notes. $z_{0}, \ell, t_{\mathrm{abs}}$ and $v_{\mathrm{cr}}$ refer to $r=10 \mathrm{AU}$.

(1) The mean energy of destructive photons $h \bar{v}$ from Eq. (5) at the bottom of the extinction layer (see Fig. 5); for photospheric and FUV photons we put $v_{\mathrm{c}}=0$ and for EUV and X-rays we integrate for $v \geq v_{\mathrm{c}}$ (Eq. (10)); (2) absorption cross section $\kappa$ per C atom at a frequency $\bar{v}$; (3) approximate mean degree of ionization of the gas in the extinction layer ; (4) extinction cross section of gas and dust at frequency $\bar{v}$ normalized to $K_{\mathrm{V}}=$ $200 \mathrm{~cm}^{2} \mathrm{~g}^{-1}$; (5) number of expelled atoms $x$ per absorption event from Eq. (14); (6) altitude of the bottom of the extinction layer in units of the scale height $H$; (7) thickness of extinction layer; (8) mean time $t_{\text {abs }}$ from Eq. (4) in which one photon is absorbed; (9) critical vertical velocity for PAH survival.

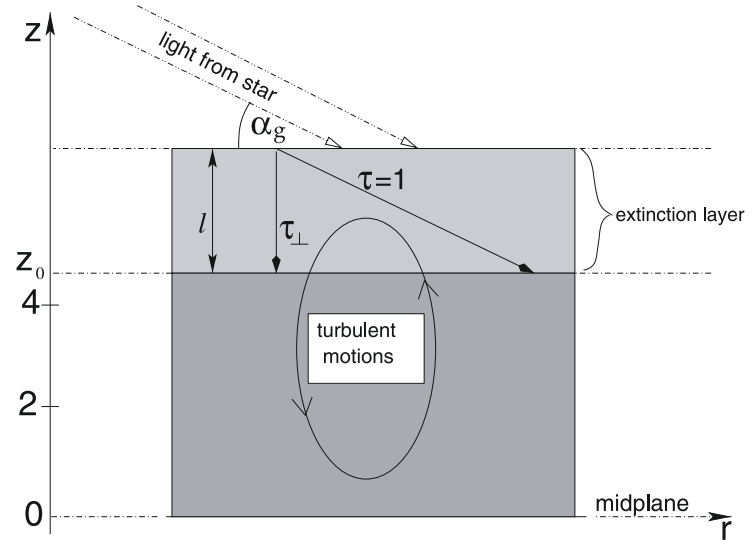

Fig. 5. Of each radiation component, $\sim 90 \%$ is absorbed in what we call the extinction layer. The optical depth from its bottom to the star is one and in the vertical direction equal to the grazing angle $\alpha_{\mathrm{g}}$. The height of its lower boundary $z_{0}$ declines with the radius, but its geometrical thickness is rather constant $(\ell \sim 0.5 \mathrm{H}$, see Fig.6). Vertical motions may replenish PAHs from below.

\section{Conditions for PAH survival}

According to Eq. (10), PAHs are destroyed if the source emits photons of an energy $h v \geq 0.1 N_{\mathrm{c}} E_{0}$, irrespective of the distance to the star or its luminosity. For $N_{\mathrm{c}}=100$, the critical photon energy is only $50 \mathrm{eV}$ (Eq. (10)). As T Tauri stars (or their jets) also radiate at X-rays and in the EUV, the surface of the disk should be devoid of PAHs unless $a$ ) the period over which hard photons are emitted is too short to destroy all PAHs; $b$ ) the PAHs are removed by vertical motions from the hard radiation before they are destroyed and there is an influx of PAHs from below; or $c$ ) PAH destruction is compensated by $\mathrm{PAH}$ formation in the surface layer. The last effect should be prevented by Coulomb repulsion (Voit 1992) in a hard photon environment where PAHs and carbon atoms are ionized.

\subsection{Destruction time}

The above PAH survival condition under $a$ ) can easily be dismissed. To estimate the time for PAH removal $t_{\text {rem }}$ by the radiation component $i$, we note that most of the radiation is absorbed on the disk surface in a sheet of vertical optical depth $\tau_{\perp}$ equal to the grazing angle $\alpha_{\mathrm{g}}$ of the incident light. We call this sheet the extinction layer (of the radiation component $i$ ) and denote its geometrical thickness $\ell_{i}$ (see Fig. 5). To first order, the PAHs in the extinction layer receive the stellar flux of Eq. (1) with $\tau_{v}=1$. If the instability criterion of Eq. (10) is fulfilled, $t_{\text {rem }}$ follows from $x N_{\gamma} t_{\text {rem }}=N_{\mathrm{c}}$, where $x$ is from Eq. (14), therefore

$t_{\mathrm{rem}}=\frac{N_{\mathrm{c}}}{x} t_{\mathrm{abs}}$.

With $t_{\text {abs }}$ from Table 2 one sees that even at $100 \mathrm{AU}, t_{\text {rem }}$ is short compared to the duration of the T Tauri phase $\left(\sim 10^{6} \mathrm{yr}\right.$, Bertout et al. 2007; Cieza et al. 2007).

\subsection{Exposure time and vertical mixing}

Next we consider the possibility that vertical motions in the disk lead to a continuous exchange between matter in the extinction layers, where almost all photons are absorbed and the PAHs are destroyed as well as the layers below where the PAHs are shielded and damaged ones are possibly rebuilt (Fig. 5). We assume that gas and dust are perfectly mixed in a mass ratio of 130:1.

In a Keplerian disk that is isothermal in $z$-direction and in hydrostatic equilibrium, the gas density changes like

$\rho(z)=\sqrt{\frac{2}{\pi}} \frac{\Sigma}{H} \mathrm{e}^{-z^{2} / 2 H^{2}}$

Here $\Sigma(r)$ is the surface density at a radius $r$, which is assumed to follow a power law,

$\Sigma(r)=\int_{0}^{\infty} \rho(z) \mathrm{d} z=\Sigma_{0}\left[\frac{r}{\mathrm{AU}}\right]^{-\gamma}$

and

$H(r)=\sqrt{k T r^{3} / G M_{*} m}$

is the scale height, $M_{*} \simeq 1 M_{\odot}$ the stellar mass and $m$ the mass of a gas molecule. For the surface density, reasonable numbers are $\gamma=1$ and $\Sigma=200 \mathrm{~g} \mathrm{~cm}^{-2}$ (Hartmann et al. 1998; Kitamura et al. 2002; Dullemond et al. 2002; Rafikov \& Colle 2006; Gorti $\&$ Hollenbach 2008), although the various estimates show considerable scatter. 
For the radial variation of the gas temperature in the opaque mid plane, $T(r)$, we also adopt a power law,

$T(r)=T_{0}\left[\frac{r}{\mathrm{AU}}\right]^{-\beta}$.

The mid plane is roughly isothermal in $z$ because the optical depth is high and the net flux zero. It is much colder than the extinction layers because it is not exposed to direct stellar heating. The radiative transfer in the disk, including the energy equation, can be solved to any desired accuracy even when the disk is very opaque (see Sect. 11.3.2 of Krügel 2006). As long as the dust in the mid plane is optically thick to its own emission, the results for $T(r)$ can be well approximated by putting in Eq. (19) $\beta=0.5$ and $T_{0} \sim 130 \mathrm{~K}$ (as also suggested by Dullemond et al. 2007b; or Chiang \& Goldreich 1997).

Each extinction layer extends vertically from some value $z_{0}$ upwards to infinity (Fig. 5). We give it a finite thickness $\ell$ by demanding that for instance $90 \%$ of the photons are absorbed between $z_{0}$ and $z_{0}+\ell$. If $v_{\perp}$ denotes the typical vertical velocity, for example, as a result of turbulence, PAHs are exposed to radiation for a time

$t_{\exp }=\frac{\ell}{v_{\perp}}$.

This is also the mean residence time of a PAH in the extinction layer. For PAHs to survive, $t_{\exp }$ must be smaller than $t_{\text {rem }}$ Eq. (15). The height $z_{0}$ follows from

$K \int_{z_{0}}^{\infty} \rho(z) \mathrm{d} z=\alpha_{\mathrm{g}}$

and $\ell$ may be estimated from the condition that only $10 \%$ of the flux is absorbed above $z_{0}+\ell$,

$K \int_{z_{0}+\ell}^{\infty} \rho(z) \mathrm{d} z=0.1 \alpha_{\mathrm{g}}$

$K$ is the mass absorption coefficient of gas and dust at the characteristic frequency of the particular radiation component (see Table 2). Because $\rho(z)$ changes rapidly, $z_{0}$ is rather insensitive both to $\alpha_{\mathrm{g}}$ as well as to $K$. For $\alpha_{\mathrm{g}} / K=10^{-8} \ldots 10^{-2}$, one obtains $z_{0}=2.6 H \ldots 5.7 H$. So in the $\mathrm{V}$ band, where absorption is only by dust ( $\left.K \simeq 200 \mathrm{~cm}^{2} \mathrm{~g}^{-1}\right)$ and for a grazing angle $\alpha_{\mathrm{g}}=3^{\circ}$, one gets $z_{0} \simeq(4 \ldots 5) H$ and $f_{\ell}=\ell / H \simeq 0.5$. The height $z_{0}$, where an extinction layer begins, and its thickness are shown in Fig. 6 ; $\ell$ is very similar for all radiation components $\left(\ell_{i} \sim H / 2, \quad i=1, \ldots, 4\right)$. EUV photons are absorbed in the highest layers, which lies about one scale height above the others. X-rays penetrate slightly deeper than photospheric or FUV photons.

From Eqs. (4), (15) and (20), one finds that vertical motions safeguard PAHs against destruction if

$v_{\perp}>v_{\mathrm{cr}}=\frac{\ell x}{t_{\mathrm{abs}} N_{\mathrm{c}}}=\frac{f_{\ell} H x}{4 \pi r^{2}} \int_{v_{\mathrm{c}}}^{\infty} \frac{L_{\nu} \mathrm{e}^{-\tau_{v}} K_{v}}{h v} \mathrm{~d} v$,

with $v_{\mathrm{c}}$ from Eq. (10). When the scale height $H$ is given by Eq. (18), $v_{\text {cr }} \propto r^{-(\beta+1) / 2}=r^{-3 / 4}$. The values of $v_{\text {cr }}$ at a distance of $10 \mathrm{AU}$ are listed in Table 2 together with other quantities relevant to PAH survival.

One expects the disk also to be turbulent. Turbulence may be driven by various processes such as shear flows in the disk (Lin \& Bodenheimer 1982), magneto-rotational instabilities (Balbus \& Harley 1991) or velocity discontinuities at places

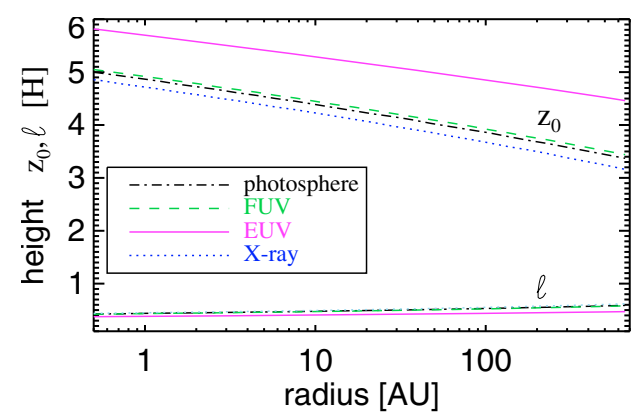

Fig. 6. The height, $z_{0}$, of the bottom of the extinction layer and its thickness $\ell$ for the four radiation components (see Fig. 5 and Eqs. (21), (22)) for a grazing angle $\alpha_{\mathrm{g}}=3^{\circ}$. Due to the high gas densities, the EUV extinction layer is practically coincident with the ionization front and lies above the extinction layer of the other components. This implies that EUV radiation is absorbed first whereas the other components penetrate deeper. Neglecting ionization by the FUV component, the gas below the $z_{0}$-line of the EUV radiation (top) is neutral.

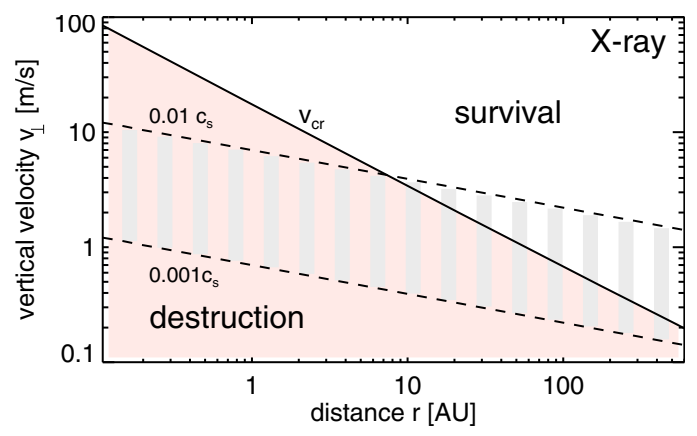

Fig. 7. The critical vertical velocity for PAH survival $v_{\text {cr }}$ after Eq. (23) with respect to X-rays as a function of distance (full line). We identify the vertical velocity $v_{\perp}$ with the turbulent velocity $v_{\mathrm{t}}$. PAHs survive when $v_{\perp}=v_{\mathrm{t}}>v_{\mathrm{cr}}$, else they are destroyed (shaded areas) by expulsion of atoms. The hatched strip between the dashed lines shows the range where $v_{\perp}$ is between $0.001 c_{\mathrm{s}}$ and $0.01 c_{\mathrm{s}}$ (where $c_{\mathrm{s}}$ is the sound velocity).

where in-falling matter (Cassen \& Mossman 1981) or outflows (Elmegreen 1978) strike the disk surface. As yet it is unclear which type of turbulence dominates. We assume that for the size of the largest eddies $\ell_{\text {ed }}$ the average turbulent velocity $v_{\mathrm{t}}$ grows linearly with the sound speed $c_{\mathrm{s}}$. Various three dimensional hydrodynamical calculations support this view (Cabot 1996; Boss 2004; Johansen \& Klahr 2005; Fromang \& Papaloizou 2006). The favored parametrization is $v_{\mathrm{t}}=\alpha^{q} c_{\mathrm{s}}$ with $q=0.5$. This choice has consequences on the eddy scale $\ell_{\mathrm{ed}}=\alpha^{1-q} H$ and the turnover time $t_{\mathrm{ed}}=\ell_{\mathrm{ed}} / v_{\mathrm{t}}=\alpha^{1-2 q} / \Omega_{\mathrm{K}}$, with the Kepler frequency $\Omega_{\mathrm{K}}=\sqrt{G M_{*} / R^{3}}$ (e.g. Dullemond \& Domink 2004). Weidenschilling \& Cuzzi (1993) use $q=1$ so that the eddy scale is about the pressure scale height, $\ell_{\mathrm{ed}}=H$ and larger than the thickness of the extinction layer, $\ell_{\mathrm{ed}}>\ell \sim H / 2$. Estimated values for $\alpha$ are in the range from 0.0001 up to 0.1 (Dullemond $\&$ Dominik 2004; Schräpler \& Henning 2004; Youdin \& Lithwick 2007). Taking $\alpha=0.01$, the eddy scale is ten times smaller for $q=0.5$ than for $q=1$, and in addition larger turbulent velocities are obtained with $v_{\mathrm{t}}=\sqrt{\alpha} c_{\mathrm{s}}$, supporting a faster transport of the PAH. Identifying $v_{\perp}$ in Eq. (23) with the turbulent velocity $v_{\mathrm{t}}$ and assuming a temperature dependence as in Eq. (19), we plot in Fig. 7 the vertical velocity $v_{\perp}$ as a function of radius. The figure also shows the critical velocity for PAH survival $v_{\text {cr }}$ with respect to X-rays. Note that the critical velocity for the X-ray radiation component is insensitive to the particular choice of $E_{0}$ and $v_{\mathrm{c}}$ 


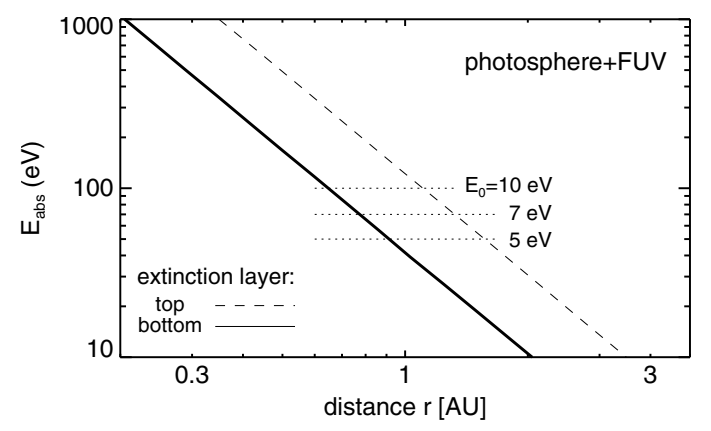

Fig. 8. The energy $E_{\text {abs }}$ (Eq. (3)), which is absorbed by a PAH of $N_{\mathrm{c}}=$ 100 carbon atoms, as a function of distance from the star. The PAH is exposed to the photospheric and FUV radiation component described in Table 1. The dashed line refers to the top of the extinction layer $(\tau=$ 0 ) and the full line to its bottom $(\tau=1)$. For an Arrhenius energy of $E_{0}=5,7$ and $10 \mathrm{eV}$, the minimum energy input $\Delta E$ (Eq. (10)) for PAH dissociation is indicated by the dotted lines.

(Eqs. (10), (23)). When $v_{\perp}=0.01 c_{\mathrm{s}}$, PAHs can survive at distances $r>10 \mathrm{AU}$; when $v_{\perp}$ is considerably smaller than $0.01 c_{\mathrm{s}}$, they cannot.

Critical velocities for PAH survival are much higher for EUV than for X-ray photons (Table 2). EUV radiation will therefore always destroy PAHs, but as depicted in Fig. 6, the EUV extinction layer is the topmost, and below it, PAHs may survive and be excited.

If PAHs are removed from the extinction layer before they are destroyed, they must be injected at the same rate from below to be detected at all. Therefore, the critical velocity can alternatively be expressed through

$\frac{1}{t_{\mathrm{rem}}} \int_{z_{0}}^{z_{0}+\ell} \rho(z) \mathrm{d} z \simeq \rho\left(z_{0}\right) v_{\mathrm{cr}}$,

which leads to similar values. We note that the mass reservoir below the extinction layer is sufficient to sustain the required mass influx $\rho v_{\text {cr }}$ over the lifetime of the disk $t_{\text {life }}$.

\subsection{PAH dissociation by soft versus hard photons}

The energy $E_{\text {abs }}$ (Eq. (3)), which is absorbed by a PAH of $N_{\mathrm{c}}=100$ carbon atoms, is shown in Fig. 8 as a function of distance from the star. The PAH is exposed to the photospheric and FUV radiation component described in Table 1, and the results are shown for the top $(\tau=0)$ and the bottom $(\tau=1)$ of the extinction layer. The minimum energy input $\Delta E$ (Eq. (10)) for PAH dissociation depends on the choice of the Arrhenius energy $E_{0}$ and is indicated for $E_{0}=5,7$ and $10 \mathrm{eV}$, respectively. In this picture, for $E_{0}=5 \mathrm{eV}$ and at the bottom of the extinction layer, PAHs are dissociated by soft photons up to 1 AU. For X-rays, however, we find that PAH destruction occurs typically at distances up to $\sim 10 \mathrm{AU}$ or greater (Fig. 7). Dissociation of PAH acts for soft (photospheric and FUV) photons on much shorter distances than for hard photons (X-ray component).

\section{Large grains}

Observations of $\mathrm{T}$ Tauri stars at millimeter wavelengths (Testi et al. 2003; Lommen et al. 2007) and in the mid-infrared (van Boekel et al. 2003; Przygodda et al. 2003; Kessler-Silacci et al. 2006; Bouwman et al. 2008; Watson et al. 2009) suggest that grains in T Tauri disks are at least ten times larger than those in the ISM. As such large grains may also be present in the top disk layer, we estimate how this would affect the stability analysis of PAHs. We first note that in case of homogeneous mixing an increase in particle size would not alter the dust-to-gas mass ratio.

Should the grains be much larger than the wavelength, the absorption coefficient per gram of dust $K_{\mathrm{d}, \lambda}$ would decrease roughly like $1 / a$, with grain size $a$, whereas the ratio $K_{\mathrm{d}, \lambda} / K_{\mathrm{d}, \mathrm{V}}$ would still roughly be given by the values in Table 2 . For hard $\mathrm{X}$-rays, on the other hand, $K_{\mathrm{d}, \lambda}$ is not sensitive to the grain size.

Therefore, if disk grains are on average ten times bigger and thus $10^{3}$ more massive than interstellar ones, we expect that the height $z_{0}$ to which the stellar radiation components can penetrate (see Fig. 6) stays the same for X-ray and also for EUV radiation, because EUV absorption is due to gas, not dust. However, optical and FUV photons will reach farther down, about half a scale height, so that there may be a thin disk layer $(\sim H / 4)$ where PAHs are shielded from X-rays and EUV photo-destruction and are excited by optical or FUV radiation.

\section{Conclusion}

Why most T Tauri stars do not exhibit PAH features while a few do, we investigate the processes which can remove PAHs from the surface layer of T Tauri disks and find out under which conditions PAHs should be present. Clearing of PAH through interaction with planets seems not an efficient process (Geers et al. 2007b), and we show that PAH under-abundance can be caused by radiative destruction. We use a fiducial model for the photon emission of the T Tauri star that includes beside the photosphere also FUV and EUV radiation and an X-ray component.

1. We introduce for each stellar radiation component the notion of an extinction layer as the place where $\sim 90 \%$ of the photons are absorbed. EUV photons are mainly absorbed by gas, $\mathrm{X}$-rays by gas and dust alike, and the photospheric and FUV component are only attenuated by dust. The extinction layers of all four components have a similar geometrical thickness, and their bottom is at a similar elevation $z_{0}$, except for the EUV extinction layer, which is situated higher up (Fig. 6).

2. PAH may be radiatively destroyed by unimolecular dissociation where one or several atoms are expelled after photon absorption.

3. Destruction by the photospheric and FUV radiation component (soft photons) increases with the strength of the radiation field and is very efficient below 1 AU.

4. Hard photons can dissociate PAHs at all distances, and their efficiency grows with the hardness of the photons. Without some counter process, all PAHs (in layers where they can be excited) would be destroyed within a time that is short compared to the lifetime of the disk.

5. Although grains in the disk surface are presumably larger than interstellar ones, the stability analysis of PAHs would not change significantly.

6. Therefore there must be some survival channel in disks where PAHs are detected. Because creation of PAHs in the extinction layer is too slow to compete with PAH destruction (Voit 1992), we suggest vertical mixing as a result of turbulence. It can replenish PAHs or remove them from the reach of hard photons.

7. For standard disk models, the minimum velocity for PAH survival is proportional to $r^{-3 / 4}$ and equals $\sim 5 \mathrm{~m} / \mathrm{s}$ at $10 \mathrm{AU}$. If turbulent velocities are proportional to the sound speed, a velocity $v_{\mathrm{t}} \geq 5 \mathrm{~m} / \mathrm{s}$ would imply $v_{\mathrm{t}} / c_{\mathrm{s}} \gtrsim 0.01$ as a PAH survival condition. Theoretical predictions for this ratio have 
a large spread, but in accordance with the observational fact that PAH features are usually absent, it seems that generally the condition is not fulfilled.

8. A higher PAH detection rate is found in Herbig Ae/Be stars. In our picture this is explained, as their destructive hard radiation component is relatively weak $\left(L_{\mathrm{x}} / L_{*} \lessgtr 10^{-7}\right.$, Preibisch et al.2006) and also because the intensity of the PAH emission from a large distance from the star is higher given their brighter optical luminosities.

Acknowledgements. We thank an anonymous referee for constructive comments on $\mathrm{PAH}$ processing.

\section{References}

Akeson, R. L., Walker, C. H., Wood, K., et al. 2005, ApJ, 622, 440 Allamandola, L. J., Tielens, A. G. G. M., \& Barker, J. R. 1989, ApJS, 71, 733

Acke, B., \& van den Ancker, M. E. 2004, A\&A, 426, 151

Balbus, S. A., \& Hawley, J. F. 1991, ApJ, 376, 214

Boss, A. P. 2004, ApJ, 610, 456

Brown, J. M., Blake, G. A., Qt, C., Dullemond, C. P., \& Wilner, D. J. 2008, ApJ, L109

Balucinska-Church, M., \& McCommon, D. 1992, ApJ, 400, 699

Bertout, C., Siess, L., \& Cabrit, S. 2007, A\&A, 473, L21

Bouwman, J., Henning, Th., \& Hillenbrand, L. A. 2008, ApJ, 683, 479

Cabot, W. 1996, ApJ, 465, 874

Calvet, N., \& Gullbring, E. 1998, ApJ, 509, 802

Cassen, P. M., \& Mossman, A. 1981, Icarus, 48, 353

Chiang, E. I., \& Goldreich, P. 1997, ApJ, 490, 368

Cieza, L., Padgettt, D. L., Stapelfeldt, K. R., et al. 2007, ApJ, 667, 328

CRC Handbook of Chemsitry \& Physics 2005 (Taylor \& Francis), 6ff

Dullemond, C. P., \& Dominik, C. 2004, A\&A, 421, 1075

Dullemond, C. P., van Zadelhoff, G. J., \& Natta, A. 2002, A\&A, 389, 464

Dullemond, C. P., Henning, Th., Visser, R., et al. 2007a, A\&A, 473, 457

Dullemond, C. P., Hollenbach, D., Kamp, I., \& D’Alessio, P. 2007b, in Protostars and Planets V, ed. B. Reipurth, D. Jewitt, \& K. Keil (Tucson: University of Arizona Press), 555

Dwek, E., \& Smith, R. K. 1996, ApJ, 459, 686

Elmegreen, B. G. 1978, Moon and Planets, 19, 21

Evans, N. J., Allen, L. E., Blake, G. A., et al. 2003, PASP, 115, 965

Fromang, S., \& Papaloizou, J. 2006, A\&A, 452, 751

Furlan, E., Hartmann, L., Calvet, N., et al. 2006, ApJS, 156, 568

Geers, V. C., Augereau, J.-C., Pontoppidan, K. M., et al. 2006, A\&A, 459, 545

Geers, V. C., van Dishoeck, E. F., Pontoppidan, K. M., et al. 2007a, A\&A, 476, 279

Geers, V. C., Pontoppidan, K. M., van Dishoeck, E. F., et al. 2007b, A\&A, 469, L35

Geers, V. C., van Dishoeck, E. F., Pontoppidan, K. M., et al. 2009, A\&A, 495, 837
Güdel, M., Skinner, S. L., \& Mel'Nikov, S. Yu. 2007, A\&A, 468, 353 Guhathakurta, P., \& Draine, B. T. 1989, ApJ, 345, 230

Gorti, U., \& Hollenbach, D. 2008, ApJ, 683, 287

Kessler-Silacci, J., Augereau, J.-C., Dullemond, C., et al. 2006, ApJ, 639, 275

Kitamura, Y., Momose, M., Yokogawa, S., et al. 2002, ApJ, 581, 357

Krügel, E. 2006, An introduction to the Physics of Interstellar Dust (IoP), Sects. 5.4 and 10.2

Johansen, A., \& Klahr, H. 2005, ApJ, 634, 1353

Judge, P. G., Solomon, S. C., \& Ayres, T. R. 2003, ApJ, 593, 534

Leach, S., Eland, J. H. D., \& Price, S. D. 1989a, J. Phys. Chem., 93, 7575

Leach, S., Eland, J. H. D., \& Price, S. D. 1989b, J. Phys. Chem., 93, 7583

Leger, A., D’Hendecourt, L., Boissel, P., \& Desert, F. X. 1989, A\&A, 213, 351

Le Page, V., Snow, T. P., \& Bierbaum, V. M. 2003, ApJ, 584, 316

Lin, D. N. C., \& Bodenheimer, P. 1982, ApJ, 262, 768

Lommen, D., Wright, C. M., Maddison, S. T., et al. 2007, A\&A, 462, 211

Meeus, G., Waters, L. B. F. M., Bouwman, J., et al. 2001, A\&A, 365, 476

Micelotta, E. R., Jones, A. P., \& Tielens, A. G. G. M. 2010a, A\&A, 510, A36

Micelotta, E. R., Jones, A. P., \& Tielens, A. G. G. M. 2010b, A\&A, 510, A37

Morrison, R., \& McCommon, D. 1983, ApJ, 270, 119

Muzerolle, J., Hartmann, L., \& Calvet, N. 1998, AJ, 116, 2965

Muzerolle, J., Calvet, N., Hartmann, L., \& D’Alessio, P. 2003, ApJ, 597, L149

Omont, A. 1986, A\&A, 166, 159

Peeters, E., Hony, S., van Kerckhoven, C., et al. 2002, A\&A, 390, 1089

Preibisch, T., Kim, Y.-C., Favata, F., et al. 2006, ApJSS, 160, 401

Przygodda, F., van Boekel, R., Abraham, P., et al. 2003, A\&A, 412, 43

Rafikov, R. R., \& de Colle, F. 2006, ApJ, 646, 275

Rapacioli, M., Calvo, F., Joblin, C., et al. 2006, A\&A, 460, 519

Ruhl, E., Price, S. D., \& Leach, S. 1989, J. Phys. Chem., 93, 6312

Salpeter, E. E. 1977, ARA\&A, 15, 267

Schräpler, R., \& Henning, Th. 2004, ApJ, 614, 960

Schutte, W. A., Tielens, A. G. G. M., \& Allamandola, L. J. 1993, ApJ, 415, 397

Siebenmorgen, R., Prusti, T., Natta, A., \& Müller, T. G. 2000, A\&A, 361, 258

Stahler, S. W., Shu, F. H., \& Taam, R. E. 1980, ApJ, 242, 226

Stelzer, B., Micela, G., Hamaguchi, K., \& Schmitt, J. H. M. M. 2006, A\&A, 457, 223

Stelzer, B., Flaccomio, E., \& Briggs, K. 2007, A\&A, 468, 463

Sterzik, M. F., \& Schmitt, H. M. M. 1997, AJ, 114 (4), 1673

Testi, L., Natta, A., Shepherd, D. S., \& Wilner, D. J. 2003, A\&A, 403, 323

Tielens, A. G. G. M. 2005, The Physics and Chemistry of the Interstellar Medium (Cambridge University Press), Sect. 6.4

Tielens, A. G. G. M. 2008, ARA\&A, 46, 289

Visser, R., Geers, V. C., Dullemond, C. P., et al. 2007, A\&A, 466, 229

Voit, G. M. 1992, MNRAS, 258, 841

van Boekel, R., Waters, L. B. F. M., Dominik, C., et al. 2003, A\&A, 400, 21

van Boekel, R., Min, M., Leinert, C., et al. 2004, Nature, 432, 479

Waelkens, C., Waters, L. B. F. M., de Graauw, M. S., et al. 1996, A\&A, 315, L245

Watson, D. M., Leisenring, J. M., Furlan, E., et al. 2009, ApJS, 180, 84

Weidenschilling, S. J., \& Cuzzi, J. N. 1993, Protostars and Planets III (A9342937 17-90), 1031

Youdin, A. N., \& Lithwick, Y. 2007, Icar, 192, 588 